\title{
Total score or subscales in scoring the acromegaly quality of life questionnaire: using novel confirmatory methods to compare scoring options
}

\section{John M Felt Jr, Sarah A Depaoli, Alberto M Pereira ${ }^{1}$, Nienke R Biermasz ${ }^{1}$ and Jitske Tiemensma}

Psychological Sciences, SSHA, University of California, Merced, 5200 North Lake Road, Merced, California 95343, USA and ${ }^{1}$ Department of Endocrinology and Metabolism, Leiden University Medical Center, Leiden,

The Netherlands
Correspondence should be addressed

to J Tiemensma

Email

jtiemensma@ucmerced.edu

\begin{abstract}
Context: Impaired quality of life (QoL) is common in patients after long-term remission of acromegaly. The acromegaly QoL (AcroQoL) is a disease-specific QoL questionnaire for patients diagnosed with acromegaly. The summed total score is the most frequently used scoring method of the AcroQoL. However, the total score does not capture all of the aspects of QoL that are outlined by the World Health Organization (WHO).

Objective: The aim of the present study was to use novel and sophisticated confirmatory methods to identify the optimal number of subscales for the AcroQoL.

Design and patients: Patients in remission from acromegaly were recruited from the Leiden University Medical Center and were asked to complete the AcroQoL (Dutch version) questionnaire $(n=72)$.

Results: The three-subscale version of the AcroQoL consisted of subscales reflecting Physical Complaints, Appearance Issues, and Personal Relations Issues related to QoL. Model fit indices (i.e., comparative fit index and root mean square error of approximation) indicated that the three-subscale version represented the data better than the total score and two-subscale models did. A $\chi^{2}$ difference test indicated that the three-subscale model was a significantly better fit than the total score and two-subscale models were $(P<0.05)$.

Conclusion: Model fit and comparison statistics indicate that the three-subscale model is a better scoring method than the total score and two-subscale versions of the AcroQoL are. The three-subscale version also better reflected the WHO's recommendation of using a multidimensional measure of QoL than the total score and two-subscale methods did. Therefore, it is recommended that values from the three-subscales of the AcroQoL be reported in future research.
\end{abstract}

\section{Introduction}

Acromegaly is a rare disease characterized by a growth hormone (GH)-secreting pituitary adenoma (1). The physical symptoms of acromegaly include frontal bossing, swelling of extremities, and swelling of soft tissue (i.e., heart, intestines, and tongue) (2) resulting from chronic $\mathrm{GH}$ overexposure. Psychological issues include negative illness perceptions (3) and a decreased quality of life (QoL)
$(1,4)$. Furthermore, patients with acromegaly are at risk for various health issues, such as arthritis, heart disease, hypertension, and diabetes mellitus (5). Acromegaly can be treated by selective removal of the adenoma via transsphenoidal surgery, followed by additional radiotherapy if needed. If surgery and/or radiotherapy is not preferred, or when this treatment is not (completely) successful,
(C) 2015 European Society of Endocrinology Printed in Great Britain
Published by Bioscientifica Ltd. 
patients can be treated with suppressive medication, such as somatostatin analogs (1) and/or GH receptor agonists (5). Upon remission of acromegaly, physical and psychological symptoms usually improve, although QoL often does not return to premorbid levels $(6,7)$.

The acromegaly QoL (AcroQoL) (8) is the first and only disease-specific QoL questionnaire for patients diagnosed with acromegaly. Webb et al. (8) developed the AcroQoL using literature searches, interviews with expert endocrinologists and patients, and exploratory statistical methods, and they found the measure to contain a threesubscale, a two-subscale, and a single total score scoring method. At present, many studies have measured QoL and changes in QoL predominantly using the single total score of the AcroQoL $(9,10,11)$. However, the World Health Organization (WHO) has recommended that QoL be treated as a multidimensional construct that, at a minimum, contains physical, psychological, and social aspects of QoL (12). There have been a few studies that examined the properties of a two-subscale and a three-subscale scoring method of the AcroQoL, but the total score is still the most frequently used scoring method $(6,8,13,14)$. However, these studies have all relied on exploratory methods to examine the different number of subscales embedded in the AcroQoL. Exploratory methods are designed for use when a measure is being created and there are few or no ideas about scale dimensionality (i.e., potential subscales), and they are often regarded as a first step in test construction.

A disadvantage of using the total score of the AcroQoL is that it collapses across the dimensions that reflect QoL. Although the AcroQoL contains questions that assess the WHO's recommended dimensions of QoL, the use of the total score makes it impossible to distinguish among those dimensions of QoL. A second limitation is that the psychometric properties of the AcroQoL have only been studied using exploratory methods. Test construction typically begins with a literature search, expert and patient interviews, and exploratory statistical methods. The next logical step in test construction is to use confirmatory statistical methods to assess the generalizability of results and to compare scoring options $(15,16)$. Previous studies $(8,13,14,17)$ have all implemented exploratory methods to assess the psychometric properties of the AcroQoL without comparing the performance of alternative scoring solutions. A subsequent step to using exploratory methods is to implement confirmatory methods, which are necessary to examine hypotheses about scale dimensionality (18). Therefore, the aim of the present study was to use sophisticated and novel confirmatory methods to determine the optimal number of subscales for the
AcroQoL. Given the WHO's recommendations, the three-subscale scoring solution was hypothesized to be the best solution for the AcroQoL.

\section{Participants and methods}

\section{Participants}

Patients in the present study were recruited by Tiemensma et al. (9), who examined associations between illness perceptions and QoL in patients with long-term remission of acromegaly. Patients were eligible for participation if they were more than 18 years of age and had been in remission, as defined by strict biochemical criteria, for at least 1 year. Patients were asked to complete the AcroQoL (Dutch version) at home and return it in a prepaid envelope. The protocol was approved by the Leiden University Medical Center Medical Ethics Committee. The long-term treatment outcome for these patients has been characterized in detail previously $(9,19)$. As per protocol, all patients had been in remission at the time of the present study for at least 1 year (mean \pm s.D. $16 \pm 10$ years). In total, 72 patients (59.7\% male; age $60.29 \pm 11.89$ years) completed the AcroQoL questionnaire.

\section{AcroQoL questionnaire}

The AcroQoL is a disease-specific QoL questionnaire designed to asses QoL in patients with acromegaly (8). The questionnaire consists of 22 items measured on a fivepoint Likert-type scale that assesses frequency of occurrence (always to never) or degree of agreement (completely agree to completely disagree) with the statements. The AcroQoL consists of questions that reflect dimensions of QoL related to Physical Complaints (e.g., 'My legs are weak'), Psychological Complaints, such as Appearance Issues (e.g., 'I feel ugly'), and problems with Personal Relations (e.g., 'I try to avoid socializing').

\section{Statistical analysis}

Data were analyzed with confirmatory factor analyses (CFA) using Mplus version 7.2 (20; Mplus, Muthén \& Muthén, Los Angeles, CA, USA). The amount of missing data was low (1.2\% missing), and the default settings in Mplus for missing data imputation and model identification were used unless otherwise noted (20). Model fit was assessed to examine the degree to which particular scoring methods of the AcroQoL scale (e.g., two-subscales, etc.) reflect the response patterns exhibited in the data. The fit of the models (i.e., the different 
scoring options) was assessed using the comparative fit index (CFI), the root mean square error of approximation (RMSEA), and the $\chi^{2}$ test. The CFI is a goodness-of-fit index, wherein values closer to one indicate acceptable fit of the model (e.g., a two-subscale model, etc.). The RMSEA is an index that reflects levels of model misfit, wherein larger values signify a worse-fitting model. Finally, a significant $\chi^{2}$ test (i.e., $P<0.05$ ) indicates a poorly fitting model (21).

The fit indices used in the present study are all absolute measures of fit that are used to assess how well the specific model is fitting the data. In contrast to the absolute fit measures, the $\chi^{2}$ difference test can be used to compare the fit of several competing models (22). Models were compared by calculating a difference score for the $\chi^{2}$ values. A significant $\chi^{2}$ difference value $(P<0.05)$ indicates that the model with the lower $\chi^{2}$ value is the better-fitting model (22).

The models examined in the present study include: i) the total score version of the AcroQoL; ii) the two-subscale version, comprised of Physical Symptoms and Psychological Symptoms; and iii) the three-subscale version, comprised of Physical Symptoms, Appearance Issues, and Personal Relations Issues. Subscale correlations were calculated, and no collinearity issues were found. Results are presented as standardized coefficients $(\beta)$ and S.E.M. Statistically significant $(P<0.05) \beta$ values larger than 0.4 are deemed to be meaningful relationships between items and subscales.

\section{Results}

\section{Single total score model}

The first scoring method assessed was the total score model, which was originally proposed by Webb et al. (8). The $\beta$ coefficients and s.e.m. for each of the items are presented in Table 1 . The fit indices for the total score model were $\mathrm{CFI}=0.804$, RMSEA $=0.182$ (90\% CI 0.168, $0.197)$, and $\chi^{2}(209)=709.274, P<0.001$. Together, the fit indices indicate a poor model fit. Each item significantly (all $P<0.05$ ) related to the total scale, although Items 11 (i.e., body size) and 16 (i.e., snoring) loaded relatively weakly ( $\beta=0.366$ and 0.321 respectively).

\section{Two-subscale scoring model}

The two-subscale model, which was also described by Webb et al. (8), was examined next. The Physical Symptoms

Table 1 Standardized $\beta$ coefficients for single total score and two-subscale models. Single total score model: $\chi^{2}$ (209) $=709.274$, $P<0.001 ; \mathrm{CFI}=0.804 ; \mathrm{RMSEA}=0.182$ (90\% Cl 0.168, 0.197). Two-subscale model: $\chi^{2}(208)=631.348, P<0.001 ; \mathrm{CFI}=0.834 ; \mathrm{RMSEA}=$ $0.168(90 \% \mathrm{Cl} 0.153,0.183)$. Correlation of Physical subscale with Psychological subscale: $r=0.722$.

\begin{tabular}{|c|c|}
\hline Item & $\beta$ coefficient \\
\hline 13 & $0.868 *$ \\
\hline 09 & $0.877^{*}$ \\
\hline 19 & $0.837^{*}$ \\
\hline 22 & $0.826^{*}$ \\
\hline 15 & $0.810^{*}$ \\
\hline 01 & $0.726^{*}$ \\
\hline 14 & $0.674^{*}$ \\
\hline 03 & $0.537 *$ \\
\hline 20 & $0.842 *$ \\
\hline 04 & $0.870 *$ \\
\hline 02 & $0.786^{*}$ \\
\hline 18 & $0.683^{*}$ \\
\hline 05 & $0.677 *$ \\
\hline 21 & $0.647 *$ \\
\hline 12 & $0.596^{*}$ \\
\hline 07 & $0.558^{*}$ \\
\hline 06 & $0.509 *$ \\
\hline 08 & $0.482 *$ \\
\hline 10 & $0.483^{*}$ \\
\hline 17 & $0.491 *$ \\
\hline 11 & $0.366^{*}$ \\
\hline 16 & $0.321 *$ \\
\hline
\end{tabular}

\begin{tabular}{|c|c|c|c|}
\hline \multicolumn{4}{|c|}{ Two-subscale AcroQoL } \\
\hline \multicolumn{2}{|c|}{ Subscale 1: Physical Symptoms } & \multicolumn{2}{|c|}{ Subscale 2: Psychological Symptoms } \\
\hline$\beta$ coefficient & S.E.M. & $\beta$ coefficient & S.E.M. \\
\hline $0.908 *$ & 0.031 & & \\
\hline $0.902 *$ & 0.031 & & \\
\hline $0.883^{*}$ & 0.044 & & \\
\hline $0.857^{*}$ & 0.033 & & \\
\hline $0.845^{*}$ & 0.034 & & \\
\hline $0.767^{*}$ & 0.048 & & \\
\hline $0.720 *$ & 0.051 & & \\
\hline \multirow[t]{15}{*}{$0.588^{*}$} & 0.082 & & \\
\hline & & $0.984 *$ & 0.049 \\
\hline & & $0.905^{*}$ & 0.037 \\
\hline & & $0.822 *$ & 0.041 \\
\hline & & $0.730 *$ & 0.056 \\
\hline & & $0.720 *$ & 0.079 \\
\hline & & $0.688^{*}$ & 0.050 \\
\hline & & $0.633^{*}$ & 0.083 \\
\hline & & $0.602 *$ & 0.068 \\
\hline & & $0.555^{*}$ & 0.100 \\
\hline & & $0.527^{*}$ & 0.082 \\
\hline & & $0.521^{*}$ & 0.077 \\
\hline & & $0.522 *$ & 0.091 \\
\hline & & $0.394 *$ & 0.093 \\
\hline & & $0.339 *$ & 0.088 \\
\hline
\end{tabular}

*Indicates significant factor loading at $P<0.05$. 
Table 2 Standardized $\beta$ coefficients for three-subscale model. $\chi^{2}(207)=529.057, P<0.001 ; \mathrm{CFI}=0.874 ; \mathrm{RMSEA}=0.147(90 \% \mathrm{CI}$ $0.132,0.163)$. Correlation of Physical subscale with Appearance subscale: $r=0.546$. Correlation of Physical subscale with Personal Relations subscale: $r=0.746$. Correlation of Personal Relations subscale with Appearance subscale: $r=0.475$.

\begin{tabular}{|c|c|c|c|c|c|c|}
\hline \multicolumn{7}{|c|}{ Three-subscale AcroQoL } \\
\hline \multirow[b]{2}{*}{ Item } & \multicolumn{2}{|c|}{ Subscale 1: Physical Symptoms } & \multicolumn{2}{|c|}{ Subscale 2: Appearance Issues } & \multicolumn{2}{|c|}{ Subscale 3: Personal Relations Issues } \\
\hline & $\beta$ coefficient & S.E.M. & $\beta$ coefficient & S.E.M. & $\beta$ coefficient & S.E.M. \\
\hline 13 & $0.905^{*}$ & 0.032 & & & & \\
\hline 09 & $0.904 *$ & 0.031 & & & & \\
\hline 19 & $0.875^{*}$ & 0.044 & & & & \\
\hline 22 & $0.859 *$ & 0.033 & & & & \\
\hline 15 & $0.849 *$ & 0.034 & & & & \\
\hline 01 & $0.771 *$ & 0.048 & & & & \\
\hline 14 & $0.722 *$ & 0.051 & & & & \\
\hline 03 & $0.591 *$ & 0.081 & & & & \\
\hline 04 & & & $0.967 *$ & 0.041 & & \\
\hline 02 & & & $0.882 *$ & 0.043 & & \\
\hline 12 & & & $0.756^{*}$ & 0.089 & & \\
\hline 07 & & & $0.697 *$ & 0.065 & & \\
\hline 17 & & & $0.618^{*}$ & 0.109 & & \\
\hline 11 & & & $0.499 *$ & 0.095 & & \\
\hline 16 & & & $0.409 *$ & 0.107 & & \\
\hline 20 & & & & & $0.977 *$ & 0.043 \\
\hline 05 & & & & & $0.977^{*}$ & 0.043 \\
\hline 18 & & & & & $0.790 *$ & 0.052 \\
\hline 21 & & & & & $0.769 *$ & 0.048 \\
\hline 06 & & & & & $0.611 *$ & 0.101 \\
\hline 08 & & & & & $0.514^{*}$ & 0.088 \\
\hline 10 & & & & & $0.489 *$ & 0.092 \\
\hline
\end{tabular}

*Indicates significant factor loading at $P<0.05$.

subscale consisted of eight items (i.e., Items 1, 3, 9, 13-15, 19, and 22), and the Psychological Symptoms subscale consisted of the remaining 14 items. The two-subscale scoring method had a slight improvement in model fit from the total score model: $\mathrm{CFI}=0.834, \mathrm{RMSEA}=0.168(90 \% \mathrm{CI}$ $0.153,0.183), \chi^{2}(208)=631.348, P<0.001$. Table 1 contains $\beta$ coefficients, S.E.M., and subscale. Each item significantly related to its corresponding subscale (all $P<0$.05). However, consistent with the total score model, Items 11 and 16 related relatively weakly to the Psychological Symptoms subscale $(\beta=0.394$ and 0.339 respectively).

\section{Three-factor model}

Finally, the three-factor model described by Webb et al. (8) was tested. The Physical Symptoms subscale consisted of eight items (i.e., Items 1, 3, 9, 13-15, 19, and 22), the Appearance Issues subscale had seven items (i.e., Items 2, $4,7,11,12,16$, and 17), and the Personal Relation Issues subscale had the remaining seven items. To avoid estimation issues tied to a negative residual variance, Item 20 was constrained to be equal to the path for Item 5 .
Upon inspection of the item content, this constraint was implemented because Item 5 (i.e., 'I avoid going out very much with friends because of my appearance') was similar in content to Item 20 (i.e., 'The physical changes produced by my illness govern my life'). Model fit improved from the total score model and the two-subscale model: $\mathrm{CFI}=0.874, \quad \mathrm{RMSEA}=0.147 \quad(90 \%$ CI $0.132,0.163)$, $\chi^{2}(207)=529.057, P<0.001$. All of the items loaded onto the subscales significantly (all $P<0.05$ ), with each item relating to its respective subscale above the 0.40 cut-off. Table 2 contains $\beta$ coefficients, s.E.M., and subscale correlations.

\section{$\chi^{2}$ difference test}

In order to compare models directly with one another, a $\chi^{2}$ difference test was used. The two-subscale model was found to be a significant improvement over the total score model, $\chi_{\text {change }}^{2}(1)=43.49, P<0.05$. The three-subscale model was found to be a significant improvement over both the total score model $\left(\chi_{\text {change }}^{2}(2)=95.040, P<0.05\right)$ and the two-subscale model $\left(\chi_{\text {change }}^{2}(1)=48.421\right.$, $P<0.05)$ of the AcroQoL. 
Table 3 Example AcroQoL total and subscale scores.

\begin{tabular}{|c|c|c|c|c|}
\hline Participant & Total & Physical Symptoms & Appearance Issues & Personal Relations Issues \\
\hline 3 & 95.45 & 100.00 & 85.71 & 100.00 \\
\hline 4 & 60.23 & 84.38 & 57.40 & 35.71 \\
\hline 15 & 75.00 & 75.00 & 96.43 & 53.57 \\
\hline 35 & 38.64 & 25.00 & 64.29 & 28.57 \\
\hline 45 & 43.18 & 53.13 & 60.71 & 14.29 \\
\hline 46 & 94.32 & 87.50 & 100.00 & 96.43 \\
\hline 47 & 78.41 & 93.75 & 89.29 & 50.00 \\
\hline 52 & 81.82 & 84.38 & 100.00 & 60.71 \\
\hline 53 & 71.59 & 84.38 & 92.86 & 35.71 \\
\hline 70 & 77.27 & 93.75 & 96.43 & 39.29 \\
\hline
\end{tabular}

Bold scores indicate when the total score and the subscales disagree.

\section{Discussion}

The present study demonstrated that the three-subscale scoring version is the best method for scoring and interpreting the AcroQoL as compared to the widely used single total score method. The present study is the first to use novel and sophisticated confirmatory methods to assess the scoring options of the AcroQoL. Confirmatory methods and model fit and comparison indices revealed that the three-subscale model better reflected the data than the twosubscale and total score did. Therefore, the three-subscale model is the optimal scoring method for the AcroQoL.

Previous exploratory studies elected to use the total score version over the two-subscale and three-subscale models of the AcroQoL $(8,17)$. Although the total score version is simple to use and exhibits desirable psychometric properties (i.e., acceptable relationships between the items and the total score), it is impossible to extract information associated with various dimensions of QoL from it. The WHO (12) recommends that measures of QoL consist of three dimensions that reflect physical, psychological, and social issues. The three-subscale scoring method of the AcroQoL, which consists of subscales that measure Physical Complaints, Social Issues, and Appearance Issues, better reflects the WHO's recommendation of a multidimensional measure of QoL as compared to the total score and two-subscale versions.

The better performance of the three-subscale model of the AcroQoL is further supported by the increased $\beta$ coefficients for Items 11 (i.e., 'Some parts of my body are too big') and 16 (i.e., 'I snore at night'). In the total score and two-subscale models, these items related weakly to the total score and the Psychological Issues subscale respectively. In the three-subscale model, Items 11 and 16 related strongly to the Appearance Issues subscale. Therefore, the three-subscale model was able to capture the item patterns of the AcroQoL better than the total score and two-subscale models were.

When using the AcroQoL, values for the AcroQoL subscales can be calculated by summing the scores within each subscale. Because the subscales contain a different number of items and are not directly comparable, the following transformation from Badia et al. (23) can be used:

$$
\frac{(X)-L}{H-L} \times 100,
$$

where $X$ is the total score of the subscale of interest, $L$ is the lowest possible score of that subscale, and $H$ is the highest possible score for that subscale. This equation transforms scores to a 0 to a 100 scale, with 100 indicating best possible QoL. The equation can also be used for the total score of the AcroQoL, because it keeps all three subscales and the total score on the same metric (23).

To further highlight the benefit of using the threesubscale version of the AcroQoL, a sample of scores for this scoring solution is presented in Table 3. For example, Patient 52 received a total AcroQoL score of 81.82 , which reflects a moderately high QoL. Further examination of the subscales reveals that Patient 52 received a relatively high score for the Physical Complaint subscale. Examination of the Personal Relations Issues subscale reveals a perfect QoL score of 100, whereas the Appearance Issues subscale revealed a score of 60.71 . Although Patient 52 exhibited a relatively high overall QoL, he or she was clearly experiencing impaired QoL with respect to his or her appearance. The relatively high scores from the other subscales masked the impairment from Appearance Issues, which suggests that the single total score did not completely capture the patient's actual QoL.

In summary, the three-subscale model, which consists of dimensions of QoL that reflect Physical Symptoms, 
Appearance Issues, and Personal Relation Issues, is the preferred scoring method of the AcroQoL. The threesubscale version exhibits superior psychometric properties as compared to the two-subscale and total score models when using confirmatory methods. Theoretically, the three-subscale model also performs better than the twosubscale and total score models because it closely follows the recommendations of the WHO regarding dimensions of QoL. Evidence for using the three-subscale version is further highlighted in a sample of scores provided in Table 3; this evidence demonstrates how the subscales can provide alternative conclusions about QoL than those that the total score may provide.

\section{Declaration of interest}

The authors declare that there is no conflict of interest that could be perceived as prejudicing the impartiality of the research reported.

\section{Funding}

This research did not receive any specific grant from any funding agency in the public, commercial or not-for-profit sector.

\section{References}

1 Ben-Shlomo A \& Melmed S. Acromegaly. Endocrinology and Metabolism Clinics of North America 200837 101-122. (doi:10.1016/j.ecl.2007.10.002)

2 Frohman L. Clinical and laboratory diagnosis of acromegaly. In Acromegaly: Diagnosis and Treatment, pp 54-67. Eds F Roelfsema \& N Biermasz, London: Future Medicine Ltd, 2012.

3 Tiemensma J, Kaptein AA, Pereira AM, Smit JW, Romijn JA \& Biermasz NR. Negative illness perceptions are associated with impaired quality of life in patients after long-term remission of Cushing's syndrome. European Journal of Endocrinology/European Federation of Endocrine Societies 2011165 527-535. (doi:10.1530/EJE-11-0307)

4 Tiemensma J. Quality of life in patients with acromegaly. In Acromegaly: Diagnosis and Treatment, pp 130-138. Eds F Roelfsema \& N Biermasz, London: Future Medicine Ltd, 2012.

5 Melmed S. Medical progress: Acromegaly. New England Journal of Medicine 2006355 2558-2573. (doi:10.1056/NEJMra062453)

6 Biermasz NR, van Thiel SW, Pereira AM, Hoftijzer HC, van Hemert AM, Smit JW, Romijn JA \& Roelfsema F. Decreased quality of life in patients with acromegaly despite long-term cure of growth hormone excess. Journal of Clinical Endocrinology and Metabolism 200489 5369-5376. (doi:10.1210/jc.2004-0669)

7 Van Aken MO, Pereira AM, Biermasz NR, van Thiel SW, Hoftijzer HC Smit JW, Roelfsema F, Lamberts SW \& Romijn JA. Quality of life in patients after long-term biochemical cure of Cushing's disease. Journal of Clinical Endocrinology and Metabolism 200590 3279-3286. (doi:10.1210/jc.2004-1375)
8 Webb SM, Prieto L, Badia X, Albareda M, Catalá M, Gaztambide S, Lucas T, Páramo C, Picó A, Lucas A et al. Acromegaly Quality of Life Questionnaire (ACROQOL) a new health-related quality of life questionnaire for patients with acromegaly: development and psychometric properties. Clinical Endocrinology 200257 251-258. (doi:10.1046/j.1365-2265.2002.01597.x)

9 Tiemensma J, Kaptein AA, Pereira AM, Smit JW, Romijn JA \& Biermasz NR. Affected illness perceptions and the association with impaired quality of life in patients with long-term remission of acromegaly. Journal of Clinical Endocrinology and Metabolism 201196 3550-3558. (doi:10.1210/jc.2011-1645)

10 Kepicoglu H, Hatipoglu E, Bulut I \& Darici E. Impact of treatment satisfaction on quality of life of patients with acromegaly. Pituitary 2013 1 1-7. (doi:10.1007/s11102-013-0544-7)

11 Mangupli R, Camperos P \& Webb SM. Biochemical and quality of life responses to octreotide-LAR in acromegaly. Pituitary 201417 495-499. (doi:10.1007/s11102-013-0533-x)

12 The WHOQOL Group. The World Health Organization quality of life assessment (WHOQOL): position paper from the World Health Organization. Social Science \& Medicine 199541 1403-1409. (doi:10.1016/0277-9536(95)00112-K)

13 Trepp R, Everts R, Stettler C, Fischli S, Allemann S, Webb SM \& Christ ER. Assessment of quality of life in patients with uncontrolled vs controlled acromegaly using the Acromegaly Quality of Life Questionnaire (AcroQoL). Clinical Endocrinology 200563 103-110. (doi:10.1111/j.1365-2265.2005.02307.x)

14 Rowles SV, Prieto L, Badia X, Shalet SM, Webb SM \& Trainer PJ. Quality of life (QOL) in patients with acromegaly is severely impaired: use of a novel measure of QOL: acromegaly quality of life questionnaire. Journal of Clinical Endocrinology and Metabolism 200590 3337-3341. (doi:10.1210/jc.2004-1565)

15 Nunnally J \& Bernstein IH. Psychometric theory. 81AD. Available at: https://scholar.google.com/scholar?hl=en\&as_sdt=0,5\&q=psychometric+theory+nunnally\#5 (accessed February 9, 2015), 1994.

16 Raykov T \& Marcoulides G. Introduction to Psychometric Theory. Available at: https://books.google.com/books?hl=en\&lr=\$id=8QDGBQAAQBA$J \& o i=$ fnd\&pg=PT5\&dq=psychometric+theory+raykov\&ots=XJ_ifKZPxG\&sig=szwiMIWsjNeqfBV-SM38mz_AR28 (accessed February 9, 2015), 2011.

17 Webb SM, Badia X \& Surinach NL. Validity and clinical applicability of the acromegaly quality of life questionnaire, AcroQoL: a 6-month prospective study. European Journal of Endocrinology/European Federation of Endocrine Societies 2006155 269-277. (doi:10.1530/eje.1.02214)

18 Furr R \& Bacgarach V. Confirmatory Factor Analysis. In Psychometrics: An Introduction, pp 331-353, Thousand Oaks: Sage, 2013.

19 Tiemensma J, Kaptein AA, Pereira AM, Smit JW, Romijn JA \& Biermasz NR. Coping strategies in patients after treatment for functioning or nonfunctioning pituitary adenomas. Journal of Clinical Endocrinology and Metabolism 201196 964-971. (doi:10.1210/jc.2010-2490)

20 Muthén L \& Muthén B. Mplus User's Guide. 7th edn. Los Angeles: Muthen \& Muthen; 1998-2014. Available at: http://www.statmodel. com/download/usersguide/Mplus user guide Ver_7_r6_web.pdf.

21 Bentler P \& Bonett D. Significance tests and goodness of fit in the analysis of covariance structures. Psychological Bulletin $1980 \mathbf{8 8}$ 588-606. (doi:10.1037/0033-2909.88.3.588)

22 Muthen B \& Muthen L. Examples: special features. In Mplus User's Guide, 7th edn, pp 443-458. 2014. Available at: http://statmodel.com/ download/usersguide/Chapter13.pdf

23 Badia X, Webb SM, Prieto L \& Lara N. Acromegaly Quality of Life Questionnaire (AcroQoL). Health and Quality of Life Outcomes 2004213. (doi:10.1186/1477-7525-2-13)

Received 25 February 2015

Revised version received 7 April 2015

Accepted 13 April 2015 